TRANSACTIONS OF THE

AMERICAN MATHEMATICAL SOCIETY

Volume 355, Number 4, Pages 1647-1668

S 0002-9947(02)03197-5

Article electronically published on November 22, 2002

\title{
THE $D$-MODULE STRUCTURE OF $R[F]$-MODULES
}

\author{
MANUEL BLICKLE
}

\begin{abstract}
Let $R$ be a regular ring, essentially of finite type over a perfect field $k$. An $R$-module $\mathcal{M}$ is called a unit $R[F]$-module if it comes equipped with an isomorphism $F^{e *} \mathcal{M} \longrightarrow \mathcal{M}$, where $F$ denotes the Frobenius map on Spec $R$, and $F^{e *}$ is the associated pullback functor. It is well known that $\mathcal{M}$ then carries a natural $D_{R}$-module structure. In this paper we investigate the relation between the unit $R[F]$-structure and the induced $D_{R}$-structure on $\mathcal{M}$. In particular, it is shown that if $k$ is algebraically closed and $\mathcal{M}$ is a simple finitely generated unit $R[F]$-module, then it is also simple as a $D_{R}$-module. An example showing the necessity of $k$ being algebraically closed is also given.
\end{abstract}

\section{INTRODUCTION}

The purpose of this article is to investigate the relationship between Frobenius actions and differential structure on modules over a regular ring $R$. Both Frobenius and $D$-module techniques were used with great success in commutative algebra. For example, in his groundbreaking work, Lyubeznik shows various finiteness properties of local cohomology modules of a regular ring, using Frobenius techniques in finite characteristic [15] (see also [13) and $D$-modules in characteristic zero [14]. In fact, these two viewpoints are somewhat reconciled by noticing that modules with a certain Frobenius action (unit $R[F]$-modules, see the definition in the next section) carry a natural $D_{R}$-module structure. A more careful analysis then shows [16], 17] that the characteristic zero and $p>0$ proofs are different manifestations of the same argument.

In this paper we further clarify this connection between the unit $R[F]$-structure and the induced $D_{R}$-structure on an $R$-module $\mathcal{M}$. Our main result implies that if $R$ is a regular ring, essentially of finite type over an algebraically closed field, then a simple, finitely generated unit $R[F]$-module $\mathcal{M}$ is $D_{R}$-simple. This is a consequence of the following theorem.

Theorem 1.1. Let $R$ be regular and essentially of finite type over the perfect field $k$. Let $\mathcal{M}$ be a finitely generated unit $R[F]$-module. Then its geometric length as an $R[F]$-module is the same as its geometric $D_{R}$-module length.

The term geometric refers to the length after tensoring with an algebraically closed extension field. In addition to correcting an inaccuracy in the literature (15], Remark 5.6a), this result plays an important part in establishing the finite characteristic analog of the Kashiwara-Brylinski intersection homology $D_{R}-$ module

Received by the editors May 10, 2002 and, in revised form, July 10, 2002.

2000 Mathematics Subject Classification. Primary 13A35,16S99,16S32.

$K e y$ words and phrases. Modules with Frobenius action, $D$-modules, $F$-modules.

(C)2002 American Mathematical Society 
3], 4. For a normal quotient $A=R / I$ of the regular ring $R$, this module, call it $\mathcal{L}(A, R)$, is constructed as the unique simple unit $R[F]$-submodule of the local cohomology $H_{I}^{c}(R)$, using Frobenius techniques. With the above result one concludes that it is also $D_{R}$-simple. Thus $\mathcal{L}(A, R)$ is a true analog of Brylinski and Kashiwara's module, which is characterized as the unique simple $D_{R}$-submodule of $H_{I}^{c}(R)$.

This paper is structured as follows: In Section 2 the basic facts about $R[F]-$ modules are briefly recalled. The main results from [15] are presented in the form in which they are needed to prove the above statements. Most proofs are omitted, since they can be found either in [15] or [3] with complete detail.

Section 3 continues with background results on $R[F]$-modules, focusing on the interaction with $D_{R}$-module theory. In this context a baby version of Frobenius descent is introduced and applied to derive some elementary $D_{R}$-structure results of unit $R[F]$-modules. Then we continue to lay out all the necessary tools for the proof of Theorem 1.1

Section 4 introduces geometric $u R[F]$-length and proves the basic results about this and related notions of length. Together with the results from Section 3, we are then able to prove Theorem 1.1.

Section 5 contains some examples showing that geometric length possibly deviates from the length as a unit $R[F]$-module in general. Consequently, the same examples also give an example of a simple, finitely generated unit $R[F]$-module which is not $D_{R}$-simple.

Acknowledgements. The results in this article are part of my dissertation at the University of Michigan. It is a great pleasure to thank my advisor, Karen Smith, for her guidance and support during my graduate studies.

Thanks go to Matt Emerton for entrusting me with early manuscripts of [9], [10], and to Brian Conrad for numerous comments on my dissertation, enhancing the content and exposition of this article greatly.

\section{2. $R[F]$-MODULES: A BRIEF RECALL}

Throughout this paper $R$ always denotes a regular ring. Mostly we will assume that $R$ is essentially of finite type over a perfect field $k$. The (absolute) Frobenius map on $R$, i.e., the ring map sending each element to its $p$ th power, is denoted by $F=F_{R}$. The associated map on $X=\operatorname{Spec} R$ we also denote by the same letter $F=F_{X}$.

If $M$ is an $R$-module, then $M^{e}$ denotes the $R-R$-bimodule, which as a left module is just $M$, but with right structure twisted by the eth iterate of the Frobenius, i.e., for $r \in R$ and $m \in M$ one has $m \cdot r=r^{p^{e}} m$. With this notation, Psekine and Szpiro's Frobenius functor is defined as $F^{*}(M)=R^{1} \otimes M$. Thinking of $F$ as a map on $\operatorname{Spec} R$, this is just the pullback functor for the Frobenius map. The following summarizes a few well-known properties of $F^{*}$ :

Proposition 2.1. (1) $F^{*}$ is right exact and commutes with direct limits.

(2) If $R$ is regular, then $F^{*}$ is exact and hence commutes with finite intersections.

\footnotetext{
${ }^{1}$ Alternatively we could use the relative Frobenius, and a similar theory would develop. To keep notation as simple as possible I chose to stick with the absolute case.
} 
Analogously, one defines the higher powers of the Frobenius map and functor. Obviously, $\left(F^{e}\right)^{*}=\left(F^{*}\right)^{e}$, and therefore we denote these higher powers of the Frobenius functor just by $F^{e *}$. Whenever $e$ is clear from the context, we denote $p^{e}$ by $q$.

Definition 2.2. An $R\left[F^{e}\right]$-module is an $R$-module $\mathcal{M}$ together with an $R$-linear map

$$
\vartheta^{e}: F^{e *} \mathcal{M}=R^{e} \otimes \mathcal{M} \longrightarrow \mathcal{M} .
$$

If $\vartheta^{e}$ is an isomorphism, then $\left(\mathcal{M}, \vartheta^{e}\right)$ is called a unit $R\left[F^{e}\right]$-module.

By adjointness of extension and restriction of scalars for the Frobenius map on Spec $R$, these maps $\vartheta^{e} \in \operatorname{Hom}\left(F^{e *} \mathcal{M}, \mathcal{M}\right)$ are in one-to-one correspondence with maps $F_{\mathcal{M}}^{e} \in \operatorname{Hom}\left(\mathcal{M}, F_{*}^{e} \mathcal{M}\right)$. Thus, alternatively, the $R\left[F^{e}\right]$-module is determined by a map $F_{\mathcal{M}}^{e}: \mathcal{M} \longrightarrow \mathcal{M}$ satisfying the $p^{e}$-linearity condition $F_{\mathcal{M}}^{e}(r m)=$ $r^{p^{e}} F_{\mathcal{M}}^{e}(m)$ for all $r \in R$ and $m \in \mathcal{M}$. The relation between the Frobenius structure $\vartheta_{\mathcal{M}}^{e}: F^{e^{*}} \mathcal{M} \longrightarrow \mathcal{M}$ and the Frobenius action $F_{\mathcal{M}}^{e}: \mathcal{M} \longrightarrow \mathcal{M}$ is illustrated by the commutation of the following diagram:

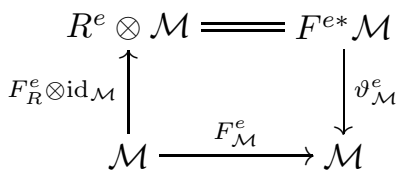

Concretely, $\vartheta^{e}(r \otimes m)=r F^{e}(m)$ and $F^{e}(m)=\vartheta^{e}(1 \otimes m)$. For convenience, the subscript " $\mathcal{M}$ " is often omitted on $\vartheta_{\mathcal{M}}^{e}$ and $F_{\mathcal{M}}^{e}$.

Now it is easy to convince oneself that such a map $F_{\mathcal{M}}^{e}$ is nothing but an action of the ring $R\left[F^{e}\right]$ on $\mathcal{M}$, where $R\left[F^{e}\right]$ denotes the (noncommutative) ring which is obtained from $R$ by adjoining the noncommutative variable $F^{e}$ to $R$ and forcing the relations $r^{p^{e}} F^{e}=F^{e} r$ of $p^{e}$-linearity. In other words, an $R\left[F^{e}\right]$-module as defined above is nothing but a module over the ring $R\left[F^{e}\right]$.

In this sense one defines the category of $R\left[F^{e}\right]$-modules, $R\left[F^{e}\right]-\bmod$, as the module category over this ring $R\left[F^{e}\right]$. As the module category over an associative ring, $R\left[F^{e}\right]$-mod is an abelian category. It is easily verified that with this definition a map of $R\left[F^{e}\right]$-modules $\left(M, \vartheta_{M}^{e}\right)$ and $\left(N, \vartheta_{N}^{e}\right)$ is a map $\varphi: M \longrightarrow N$ of $R$-modules such that

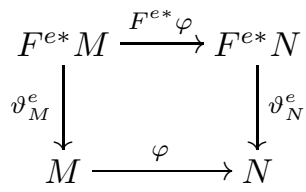

commutes. The category of unit $R\left[F^{e}\right]$-modules, $u R\left[F^{e}\right]$-mod, is the full subcategory whose objects are those $R\left[F^{e}\right]$-modules that are unit. Using that for a regular ring the Frobenius functor $F^{e^{*}}$ is exact, it follows that $u R\left[F^{e}\right]-\bmod$ is also an abelian category (see [3, Chapter 2] for details).

For all $r>0$ one has an inclusion of rings, $R\left[F^{e r}\right] \subseteq R\left[F^{e}\right]$, yielding the reverse inclusion $R\left[F^{e}\right]-\bmod \subseteq R\left[F^{e r}\right]-\bmod$ of categories. If $\left(\mathcal{M}, \vartheta^{e}\right)$ is an $R\left[F^{e}\right]-$ module, then the Frobenius structure of $\mathcal{M}$, viewed as an $R\left[F^{e r}\right]$-module, is defined inductively as $\vartheta^{e r}=\vartheta^{e(r-1)} F^{e(r-1) *}\left(\vartheta^{e}\right)$. It follows that the categories $\left\{R\left[F^{e}\right]-\bmod \mid e \in \mathbb{N}\right\}$ form a directed system whose limit is denoted by $R[F]$-mod. Thus an $R[F]$-module is an $R\left[F^{e}\right]$-module for some $e$, not necessarily explicitly 
specified 2 It is easily checked that the category of $R[F]$-modules is also abelian. Analogously one obtains the category of unit $R[F]-$ modules as the directed limit of the categories of unit $R\left[F^{e}\right]$-modules for various $e$. Again, $u R[F]-\bmod$ is abelian.

Example 2.3. The map $R^{e} \otimes R \longrightarrow R$ sending $r^{\prime} \otimes r \mapsto r^{\prime} r^{q}$ makes $R$ into a unit $R\left[F^{e}\right]$-module.

Similarly, a localization $S^{-1} R$ of $R$ is a unit $R\left[F^{e}\right]$-module via the map $r^{\prime} \otimes \frac{r}{s} \mapsto$ $\frac{r^{\prime} r^{q}}{s^{q}}$. The inverse of this map is given by sending $\frac{r}{s}$ to $r s^{q-1} \otimes \frac{1}{s}$.

2.0.1. Functors and base change. Let $G$ be an additive functor from $R-\bmod$ to $A$-mod, where $A$ is also a ring of finite characteristic $p$. If $G$ commutes with the Frobenius functor (i.e., we have a natural transformation of functors $F_{A}^{e *} \circ G \cong$ $\left.G \circ F_{R}^{e *}\right)$, then $G$ naturally extends to a functor from $R\left[F^{e}\right]-\bmod$ to $A\left[F^{e}\right]$-mod. Indeed, if $\left(M, \vartheta^{e}\right)$ is a (unit) $R\left[F^{e}\right]$-module, then

$$
F^{e *}(G(M)) \cong G\left(F^{e *}((M)) \stackrel{G\left(\vartheta^{e}\right)}{\longrightarrow} G(M)\right.
$$

defines a (unit) $A\left[F^{e}\right]$-module structure on $G(M)$. The first map is, of course, the natural isomorphism of functors which was assumed. To verify that this definition is functorial is straightforward. Since $G$ commuting with $F^{e *}$ implies that $G$ also commutes with the higher powers of $F^{e *}$, the construction just described is indeed a functor on (unit) $R[F]$-modules, i.e., compatible with the inclusion of categories $R\left[F^{e}\right]-\bmod \subseteq R\left[F^{e r}\right]-\bmod$ for $r>0$.

It follows that for a map of rings $R \longrightarrow A$, tensoring with $A$ over $R$ is a functor from (unit) $R[F]$-modules to (unit) $A[F]$-modules. All we need for this is the natural isomorphism of $A-R$-bimodules

$$
A^{e} \otimes_{A} A \cong A^{e} \cong A \otimes_{R} R^{e} .
$$

Concretely, if $\left(\mathcal{M}, \vartheta^{e}\right)$ is an $R[F]$-module, then the $A[F]$-structure on $A \otimes \mathcal{M}$ is given by $a^{\prime} \otimes a \otimes m \mapsto a^{\prime} a^{q} \vartheta^{e}(1 \otimes m)$ (equivalently, the Frobenius action is defined by $\left.F_{A \otimes \mathcal{M}}^{e}=F_{A}^{e} \otimes F_{\mathcal{M}}^{e}\right)$.

2.0.2. Notational conventions and Frobenius. If $M$ is an $R$-submodule of the $R[F]-$ module $\left(\mathcal{M}, \vartheta^{e}, F^{e}\right)$, then $F^{e *} M$ is a submodule of $F^{e *} \mathcal{M}$. Its image in $\mathcal{M}$ under $\vartheta^{e}$ we denote by $R F^{e}(M)=\vartheta^{e}\left(F^{e *} M\right)$, or briefly just by $F^{e}(M)$. Indeed, by diagram (1) $R F^{e}(M)$ is the $R$-submodule of $\mathcal{M}$ generated by the elements $F^{e}(m)$ for $m \in M$. If $\mathcal{M}$ is unit, then $F^{e *} M$ is, via $\vartheta^{e}$, isomorphic to $R F^{e}(M)$. More specifically, on the category of $R$-submodules of $\mathcal{M}$, the two functors $F^{e *}$ and $R F^{e}\left(\_\right)$are isomorphic since $R F^{e}\left(\_\right)=\vartheta^{e} \circ F^{e *}\left(\_\right)$. As it turns out, working with the more explicit $F^{e}\left({ }_{Z}\right)=R F^{e}\left({ }_{-}\right)$makes many arguments more transparent, since one never leaves the ambient module $\mathcal{M}$. This viewpoint will be used frequently without further mention.

2.1. Finitely generated unit $R[F]$-modules. The strength of $R[F]$-modules lies in bringing some sort of finiteness to, a priori, infinitely generated objects, such as local cohomology modules. This is simply achieved by enlarging the ring from $R$ to the quite big, noncommutative ring $R\left[F^{e}\right]$. Exactly the same happens when

\footnotetext{
${ }^{2}$ In $\left[3\right.$ these categories are denoted by $R\left[F^{\infty}\right]-\bmod$ and $u R\left[F^{\infty}\right]-\bmod$ respectively. The idea behind the new notation here is that omission of an exponent on $F$ means an unspecified exponent and not exponent one. Thus $R[F]-\bmod$ seems more adequate to denote this category, as well as being notationally much more convenient.
} 
$D_{R}$-module techniques are used; there it is the ring of differential operators which introduces additional structure.

Definition 2.4. An $R[F]$-module $\left(\mathcal{M}, \vartheta^{e}\right)$ is called finitely generated if it is a finitely generated module over the ring $R\left[F^{e}\right]$.

Note that, if $\mathcal{M}$ is generated by some finite subset $S \subseteq \mathcal{M}$ as an $R\left[F^{e}\right]$-module, then, as an $R\left[F^{e r}\right]$-module, $\mathcal{M}$ is generated by the finite set $S \cup F^{e}(S) \cup \cdots \cup$ $F^{e(r-1)}(S)$. Thus the finitely generated $R\left[F^{e}\right]$-modules are a subset of the finitely generated $R\left[F^{e r}\right]$-modules. Therefore, the category of finitely generated $R[F]-$ modules is, again, just the limit of the categories of finitely generated $R\left[F^{e}\right]_{-}$ modules for various $e$.

We recall some basic facts from Lyubeznik, but offer a slightly different viewpoint. The basic construction is that of a generator of a unit $R[F]$-module. Let $\varphi: M \longrightarrow F^{e *} M$ be an $R$-linear map. Consider the directed system one obtains by taking higher Frobenius powers of this map. The limit one obtains

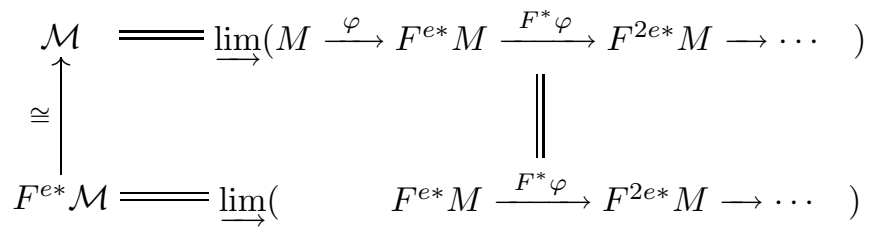

carries a natural unit $R\left[F^{e}\right]$-module structure as indicated, taking into consideration that $F^{e *}$ commutes with direct limits.

If a unit $R[F]$-module $\left(\mathcal{M}, \vartheta^{e}\right)$ arises in such a fashion, one calls $\varphi$ a generator of $\left(\mathcal{M}, \vartheta^{e}\right)$. If $M$ is finitely generated and $\varphi$ is injective, then $M$ is called a root of $M$. In this case one identifies $M$ with its isomorphic image in $\mathcal{M}=\lim F^{e r *} M$. Thus a root of a unit $R[F]$-module $\mathcal{M}$ is a finitely generated $R$-submodule $M$ such that $M \subseteq R F^{e}(M)$ and $\mathcal{M}=\bigcup_{r} R F^{e r}(M)=R\left[F^{e}\right] M$.

A key observation is the following proposition [9].

Proposition 2.5. The unit $R[F]-\operatorname{module}\left(\mathcal{M}, \vartheta^{e}\right)$ is finitely generated if and only if $\mathcal{M}$ has a root.

Proof. The "only if" direction is easy, since a finite set of $R$-module generators of a root $M$ of $\mathcal{M}$ generates $\mathcal{M}$ as an $R\left[F^{e}\right]$-module.

Conversely, let $M^{\prime}$ be the $R$-module generated by some finitely many $R\left[F^{e}\right]-$ module generators of $\mathcal{M}$. In other words,

$$
R\left[F^{e}\right] M^{\prime}=\sum_{n=0}^{\infty} F^{n e}\left(M^{\prime}\right)=\mathcal{M} .
$$

Since $\vartheta^{e}$ is an isomorphism, $\mathcal{M}=\vartheta\left(R^{e} \otimes \mathcal{M}\right)=F^{e}(\mathcal{M})$. Applying $F^{e}$ to (2), we get

$$
\mathcal{M}=F^{e}(\mathcal{M})=F^{e}\left(\sum_{n=0}^{\infty} F^{n e}\left(M^{\prime}\right)\right)=\sum_{n=1}^{\infty} F^{n e}\left(M^{\prime}\right) .
$$

Since $M^{\prime}$ was finitely generated, it is contained in a finite part of the above sum, say $M^{\prime} \subseteq \sum_{n=1}^{m} F^{n e}\left(M^{\prime}\right)$. Now we set $M=\sum_{n=0}^{m-1} F^{n e}\left(M^{\prime}\right)$, and we see right away that $M \subseteq F^{e}(M)$. Repeated application of $F^{e}\left(\_\right)$yields the following sequence of inclusions:

$$
M \subseteq F^{e}(M) \subseteq F^{2 e}(M) \subseteq F^{3 e}(M) \subseteq \cdots,
$$


whose union is $\mathcal{M}$ since $M$ contains $M^{\prime}$. Thus $M$ is the desired root of $\mathcal{M}$.

This proposition allows for easy proofs of the following theorem of [15, Theorem 2.8:

Theorem 2.6. The category of finitely generated unit $R[F]$-modules is an abelian subcategory of the category of $R[F]$-modules that is closed under extensions.

All finitely generated unit $R[F]$-modules have the ascending chain condition in the category of unit $R[F]$-modules.

Proof. Everything except the fact that the kernels are finitely generated easily follows using either the exactness of $F^{e^{*}}$ or basic facts about categories of finitely generated modules over an associative ring (they are abelian and closed under extensions). Thus it remains to show that a unit $R\left[F^{e}\right]$-submodule $\mathcal{N}$ of a finitely generated unit $R\left[F^{e}\right]$-module $\mathcal{M}$ is also finitely generated. To see this, let $M$ be a root of $\mathcal{M}$, i.e., $M \subseteq R F^{e}(M)$ and $M=R\left[F^{e}\right] M$. Then

$$
M \cap \mathcal{N} \subseteq R F^{e}(M) \cap \mathcal{N}=R F^{e}(M) \cap R F^{e}(\mathcal{N})=R F^{e}(M \cap \mathcal{N})
$$

and $R\left[F^{e}\right](M \cap \mathcal{N})=R\left[F^{e}\right] M \cap R\left[F^{e}\right] \mathcal{N}=\mathcal{M} \cap \mathcal{N}=\mathcal{N}$. Thus $M \cap \mathcal{N}$ is a root of $\mathcal{N}$, and therefore $\mathcal{N}$ is finitely generated as an $R\left[F^{e}\right]$-module. In this argument the fact (Proposition 2.1) that $R F\left(\_\right)$commutes with finite intersection was used repeatedly.

To see that a finitely generated unit $R[F]$-module has ACC, note that we just showed that a unit $R[F]$-submodule $\mathcal{N} \subseteq \mathcal{M}$ is determined by its root $N$, the intersection of $\mathcal{N}$ with a fixed root $M$ of $\mathcal{M}$. Thus increasing chains of unit $R[F]-$ submodules of $\mathcal{M}$ correspond, by intersecting with the root $M$, to certain chains of submodules of $M$. The latter stabilize because $M$ is finitely generated as an $R$-module, and thus so do the former.

Already the fact that $u R[F]-\bmod$ has ACC is very useful for proving finiteness statements for local cohomology modules, such as the finiteness of the set of associated primes or the finiteness of the Bass numbers. But even more is true. The following is Theorem 3.2 of [15]; we recall it here without proof.

Theorem 2.7. Let $R$ be a regular finitely generated algebra over a regular local ring. Then, in the category of unit $R\left[F^{e}\right]$-modules, the finitely generated ones have finite length.

It is an open problem whether this theorem is true without the assumptions on $R$.

\section{Frobenius aCtion And differential structure}

The connection between unit $R[F]$-modules and differential operators originates in the following description of the ring of differential operators over a ring of finite characteristic.

Proposition 3.1. Let $R$ be a finitely generated algebra over its subring $R^{p}$ of pth powers. Then

$$
D_{R}=\bigcup \operatorname{End}_{R^{p^{e}}}(R)
$$

is the ring of $k$-linear differential operators on $R$. 
For our purpose this proposition can be taken as the definition of the ring of differential operators, therefore eliminating the need of reviewing its proof, which can be found in [21]. The sets $\operatorname{End}_{R^{p^{e}}}(R)$ are called the differential operators of level $e$ and are denoted by $D_{R}^{(e)}$. The interested reader will find a gentle introduction to differential operators in finite characteristic in [3] for the ultimate account see [1], 2].

With this description of the ring of differential operators the action of $D_{R}$ on a unit $R[F]$-module becomes fairly straightforward. First note that $\operatorname{End}_{R^{p^{e}}}(R)$ can be identified with $\operatorname{End}_{\bmod -R}\left(R^{e}\right)$, the right $R$-module endomorphisms of $R^{e}$. For big enough $r$, a given differential operator $\delta$ lies in the set $D_{R}^{(e r)}=\operatorname{End}_{\bmod -R}\left(R^{e r}\right)$. For such $r$ the action of $\delta$ on a unit $R\left[F^{e}\right]$-module $\left(M, \vartheta^{e}\right)$ is given by the dashed arrow of the following diagram:

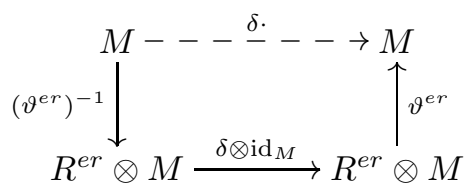

To check that this is independent of the chosen $r$ is straightforward. This also shows that one gets the same $D_{R}$-structure whether one considers $\mathcal{M}$ as a unit $R\left[F^{e}\right]-$ module or a unit $R\left[F^{e r}\right]$-module, for some $r>0$. This implies that the process of equipping a unit $R\left[F^{e}\right]$-module with an underlying $D_{R}$-structure is compatible with the inclusion of categories $u R\left[F^{e}\right]-\bmod \subseteq u R\left[F^{e r}\right]$, thus is well defined as a functor from $u R[F]-\bmod$ to $D_{R}-\bmod$. The next lemma summarizes some properties of this $D_{R}$-structure.

Lemma 3.2. (1) The $D_{R}$-structure on $R$ induced by the canonical unit $R[F]-$ structure is the canonical $D_{R}$-structure on $R$.

(2) The described process of equipping a unit $R[F]$-module with a $D_{R}$-module structure is an exact functor which commutes with localization.

For more details and proofs of these (and the following) statements, the reader should refer to [3], Chapter 3, or [15], Section 5 .

3.1. Frobenius descent. The interplay between Frobenius structures and $D_{R^{-}}$ modules becomes apparent by the simple, but powerful, fact that the Frobenius functor is an equivalence of the category of $D_{R}$-modules with itself. Ultimately, this is a consequence of the description of $D_{R}$ as in Proposition 3.1 together with the observation that all the endomorphism $\operatorname{rings} D_{R}^{(e)}=\operatorname{End}_{\bmod -R}\left(R^{e}\right)$ are Morita equivalent to $R$ itself. This, on the other hand, is easily seen, since, for a regular $R$, $R^{e}$ is locally a free right $R$-module and therefore $\operatorname{End}_{\bmod -R}\left(R^{e}\right)$ is just a matrix ring over $R$, thus Morita equivalent to $R$. Then one checks that this Morita equivalence between $R$ and $\operatorname{End}_{\bmod -R}\left(R^{e}\right)$ is, in fact, given by the Frobenius functor $F^{e^{*}} \cong$ $R^{e} \otimes \ldots$. One obtains the following theorem.

Theorem 3.3 (Frobenius descent). Let $R$ be regular and $F$-finite. The Frobenius functor $F^{e^{*}}$ is an equivalence of categories between the category of $R$-modules and that of $D_{R}^{(e)}-$ modules. The functor inverse to $F^{e *}$ is given by

$$
T^{e *} \stackrel{\text { def }}{=} \operatorname{Hom}_{\bmod -R}\left(R, R^{e}\right) \otimes_{D_{R}^{(e)}} \text {. }
$$


The functors $F^{e^{*}}$ and $T^{e *}$ induce an auto-equivalence of the category of $D_{R^{-}}$ modules.

This result appears, much generalized, in Berthelot 2 under the name of Frobenius descent. Similar versions are used by S. P. Smith [20], [19, B. Haastert [11, [12] and R. Bøgvad [5]; also Lyubeznik implicitly uses Frobenius descent to prove the following theorem.

Theorem 3.4 ([15], Theorem 5.6). Let $R$ be regular and $F$-finite. Then, a finitely generated unit $R\left[F^{e}\right]$-module that has finite length as a unit $R\left[F^{e}\right]$-module also has finite length as a $D_{R}$-module.

In particular, if $R$ is a finitely generated algebra over a regular local ring, then all finitely generated unit $R\left[F^{e}\right]$-modules have finite $D_{R}$-module length.

For the proof, please refer to [15]. In the next section part of the argument will be given to see explicitly how Frobenius descent enters.

If $\mathcal{M}$ is a $D_{R^{-}}$module, the $D_{R^{-}}$structure that $F^{e *} \mathcal{M}$ obtains via Frobenius descent is described as follows. Since $\mathcal{M}$ is a $D_{R}^{(e)}$-module for all $e$, we can write, by Frobenius descent, $\mathcal{M} \cong F^{e *} T^{{ }^{*}} \mathcal{M}$, where $T^{e *} \mathcal{M}$ is an $R$-module. Thus $F^{*} \mathcal{M} \cong F^{(e+1) *} T^{e *} \mathcal{M}$ obtains a $D_{R}^{(e+1)}$-module structure by having $\delta$ act via $\delta \otimes \operatorname{id}_{T^{e *} \mathcal{M}}$. One can check (similarly as one checks the well-definedness of the induced $D_{R}$-action from a unit $R[F]$-module) that the $D_{R}^{(e)}$-structures on $F^{*} \mathcal{M}$ that one obtains in this way for all $e$ are compatible and define a $D_{R}$-structure on $\mathcal{M}$.

3.1.1. Frobenius descent for unit $R[F]$-modules. As we have noted before, for an $R$-submodule $N$ of a unit $R\left[F^{e}\right]$-module $\left(\mathcal{M}, \vartheta^{e}, F^{e}\right)$, the image $F^{e *} N$ of $N$ under the Frobenius functor finds a concrete description as the $R$-submodule $F^{e}(N)$ of $\mathcal{M}$. Similarly, for a $D_{R}$-submodule $\mathcal{N}$ of $\mathcal{M}$ the $D_{R}$-module $T^{e *} \mathcal{N}$ can be realized as a $D_{R}$-submodule $T^{e}(\mathcal{N})$ of $\mathcal{M}$ as follows: On the category of $D_{R}$-submodules of $\mathcal{M}$, the functor $T_{\mathcal{M} \_}^{e *}$ can be identified with $T_{\mathcal{M}}^{e}\left(\_\right) \stackrel{\text { def }}{=} T_{\mathcal{M}}^{e *}\left(\vartheta^{-1}\left(\_\right)\right)$. Together with the natural identification $T^{e^{*}} F^{e^{*}} \mathcal{M} \cong \mathcal{M}$ this makes $T^{e}(\mathcal{N})$ a submodule of $\mathcal{M}$. Clearly, $F^{e} \circ T^{e}=T^{e} \circ F^{e}$ are the identity functor on $D_{R}$-submodules of $\mathcal{M}$. A more careful investigation shows that $T_{\mathcal{M}}^{e}(\mathcal{N})=\left(F_{\mathcal{M}}^{e}\right)^{-1}(\mathcal{N})$ as submodules of $\mathcal{M}$. Since we don't need this description in what follows, its proof is omitted and the reader is referred to [3], Chapter 3 , for details.

When working with $D_{R}$-submodules of a fixed unit $R[F]$-module, it is advantageous to use the un-starred variants of the functors $T^{e^{*}}$ and $F^{e *}$, since this makes the arguments much more transparent.

3.2. $D_{R}[F]-$ modules. If $\left(\mathcal{M}, \vartheta^{e}\right)$ is a unit $R\left[F^{e}\right]$-module, then $F^{e *} \mathcal{M}$ carries a natural $D_{R}$-structure by Frobenius descent, as well as by being a unit $R\left[F^{e}\right]-$ module $\left(F^{e *} \mathcal{M}, F^{e *}\left(\vartheta^{e}\right)\right)$. To show that these two $D_{R^{-} \text {structures are the same }}$ comes down to checking that $\vartheta^{e}$ is $D_{R}$-linear with respect to the $D_{R}$-structure on $F^{e *} \mathcal{M}$ coming from Frobenius descent. This motivates us to define a $D_{R}\left[F^{e}\right]_{-}$ module in analogy with $R\left[F^{e}\right]$-modules as a $D_{R}$-module $\mathcal{M}$ together with a $D_{R^{-}}$ linear map $\vartheta^{e}: F^{e *} \mathcal{M} \longrightarrow \mathcal{M}$. Of course, the $D_{R^{-}}$structure on $F^{e *} \mathcal{M}$ is the one coming from Frobenius descent.

Definition 3.5. A $D_{R}\left[F^{e}\right]$-module is a $D_{R}$-module $\mathcal{M}$ together with a $D_{R}$-linear map

$$
\vartheta_{\mathcal{M}}^{e}: F_{R}^{e *} \mathcal{M} \longrightarrow \mathcal{M}
$$


In other words, a $D_{R}\left[F^{e}\right]-$ module is an $R\left[F^{e}\right]$-module $\left(\mathcal{M}, \vartheta^{e}\right)$ that carries a $D_{R^{-}}$ structure such that $\vartheta^{e}$ is $D_{R}$-linear. $\left(\mathcal{M}, \vartheta^{e}\right)$ is called unit if $\vartheta^{e}$ is an isomorphism.

As just elaborated, unit $R\left[F^{e}\right]$-modules are $D_{R}\left[F^{e}\right]$-modules. Conversely, by forgetting the $D_{R}$-structure, every unit $D_{R}\left[F^{e}\right]$-module is a unit $R\left[F^{e}\right]$-module. Thus, for the unit case this does not lead to anything new. One reason for working with the more complicated category of $D_{R}\left[F^{e}\right]$-modules is the following proposition.

Proposition 3.6. Let $R$ be regular and $F$-finite. Then a $D_{R}\left[F^{e}\right]$-submodule of a finitely generated unit $R\left[F^{e}\right]$-module is also unit (and finitely generated).

Note that the equivalent statement for $R\left[F^{e}\right]$-modules is not true. The example of an ideal $I$ of $R$ that is an $R\left[F^{e}\right]$-submodule but not unit shows this nicely.

Proof of 3.6. We have to show that a $D_{R}\left[F^{e}\right]$-submodule of a finitely generated unit $R\left[F^{e}\right]$-module is also unit. If $\mathcal{N}$ is such an $F^{e}$-stable $D_{R}$-submodule of a unit $R\left[F^{e}\right]$-module $\mathcal{M}$, then $F^{e}(\mathcal{N}) \subseteq \mathcal{N}$. Applying $T^{e}$ and using its defining property as the inverse functor of $F^{e}$, we see that $\mathcal{N} \subseteq T^{e}(\mathcal{N})$. Iterating, we get an increasing chain of $R$-modules

$$
\mathcal{N} \subseteq T^{e}(\mathcal{N}) \subseteq T^{2 e}(\mathcal{N}) \subseteq \ldots
$$

Intersecting this chain with a root $M$ of $\mathcal{M}$ yields a chain of submodules of $M$ which, since $M$ is a finitely generated $R$-module, must stabilize. Let $N \stackrel{\text { def }}{=} T^{e r}(\mathcal{N}) \cap M=$ $T^{e(r+1)}(\mathcal{N}) \cap M=\ldots$ be the stable member. Using $F^{e r}\left(T^{e r}(\mathcal{N})\right)=\mathcal{N}$, we see that $F^{e}(N)=F^{e}\left(T^{e(r+1)}(\mathcal{N}) \cap M\right)=T^{e r}(\mathcal{N}) \cap F^{e}(M) \supseteq T^{e r}(\mathcal{N}) \cap M=N$. Applying $F^{e r}$ for all $r$ to this inclusion, we get another increasing sequence:

$$
N \subseteq F^{e}(N) \subseteq F^{2 e}(N) \subseteq \ldots
$$

Let $\mathcal{L}$ be its limit. Since $\mathcal{L}$ arises as the increasing union of the Frobenius powers of a single submodule, it is obviously a unit submodule of $\mathcal{N}$, i.e., $F^{e}(\mathcal{L})=\mathcal{L} \subseteq \mathcal{N}$. For the converse inclusion let $n \in \mathcal{N}$. For all sufficiently large $r \geq 0$ we have $n \in F^{e r}(M)$. For such $r$ also $N=T^{e r}(\mathcal{N}) \cap M$, and thus $F^{e r}(N)=\overline{\mathcal{N}} \cap F^{e r}(M)$. Thus $n \in F^{e r}(N)$, and therefore $n \in \mathcal{L}$, since $\mathcal{L}$ is the increasing union of all $F^{e r}(N)$.

Remark 3.7. Analogously to the case of $R\left[F^{e}\right]$-modules, one can show that $D_{R}\left[F^{e}\right]_{-}$ modules are just modules over an appropriate ring $D_{R}\left[F^{e}\right]$. In the case that the $\operatorname{ring} R\left[F^{e}\right]$ is in fact a subring of $\operatorname{End}_{k}(R)$ we can think of this $\operatorname{ring} D_{R}\left[F^{e}\right]$ as the subring of $\operatorname{End}_{k}(R)$ generated by $R\left[F^{e}\right]$ and $D_{R}$. In general one can define the ring $D_{R}\left[F^{e}\right]$ to be $R\left[F^{e}\right] \otimes_{R} D_{R}$, and then equip this tensor product with an appropriate ring structure. This is done in [10], where many other interesting properties in this context are shown. For example, they show the following (at first) surprising result:

Proposition 3.8. Let $R$ be a regular ring essentially of finite type over a perfect field $k$. Let $M$ be a unit $R\left[F^{e}\right]$-module. The following are equivalent.

(1) $M$ is finitely generated as a $D_{R}\left[F^{e}\right]-$ module.

(2) $M$ is finitely generated as an $R\left[F^{e}\right]-$ module.

(3) $M$ is finitely generated as a $D_{R}$-module.

The proof of this is a clever application of Frobenius descent together with [15], Theorem 5.6. 
3.3. $D_{R}$-semisimple unit $R[F]-$ modules. An object $\mathcal{M}$ in an abelian category is called semisimple if every subobject is a direct summand. If $\mathcal{M}$ also has finite length (in the category), this is equivalent to every simple subobject being a direct summand. In turn, this can be shown to be the same as $\mathcal{M}$ decomposing into a finite direct sum of simple objects. The first result indicating the importance of the notion of semisimplicity in the context of unit $R[F]$-modules and $D_{R}$-modules is the following proposition, which is implicit in the proof of [15, Theorem 5.6.

Proposition 3.9. Let $R$ be regular and $F$-finite. Let $\left(\mathcal{M}, \vartheta^{e}\right)$ be a simple finitely generated unit $R\left[F^{e}\right]-$ module. Then $\mathcal{M}$ is semisimple as a $D_{R}$-module.

Proof. We show that every simple $D_{R}$-submodule $\mathcal{N}$ of $\mathcal{M}$ is a direct $D_{R}$-module summand. By Frobenius descent, $F^{e} \mathcal{N}$ is a simple $D_{R}$-submodule of $\mathcal{M}$. Repeatedly applying $F^{e}\left(\_\right)$, we get a series $\left\{F^{r e} \mathcal{N}\right\}$ of simple $D_{R}$-submodules of $M$. Let $r$ be the first time such that the intersection

$$
\left(\mathcal{N}+F^{e} \mathcal{N}+F^{2 e} \mathcal{N}+\ldots+F^{r e} \mathcal{N}\right) \cap F^{(r+1) e} \mathcal{N}
$$

is nonempty. Since $\mathcal{M}$ has finite $D_{R}$-module length by Theorem 3.4, such $r$ exists. For this $r$, the sum on the left is direct; in particular, $\mathcal{N}$ is a direct summand of $\mathcal{M}^{\prime} \stackrel{\text { def }}{=} \mathcal{N} \oplus F^{e} \mathcal{N} \oplus F^{2 e} \mathcal{N} \oplus \ldots \oplus F^{r e} \mathcal{N}$. Furthermore, $F^{(r+1) e} \mathcal{N}$ is contained in $\mathcal{M}^{\prime}$, by simplicity of $F^{(r+1) e} \mathcal{N}$. Then $F^{e} \mathcal{M}^{\prime} \subseteq \mathcal{M}^{\prime}$, and therefore $\mathcal{M}^{\prime}$ is a $D_{R^{-}}$ and $R\left[F^{e}\right]$-submodule of $\mathcal{M}^{\prime}$. By Proposition $3.6, \mathcal{M}^{\prime}$ is a unit $R\left[F^{e}\right]$-submodule of $\mathcal{M}$. By simplicity of $\mathcal{M}$ as a unit $R\left[F^{e}\right]$-module it follows that $\mathcal{M}^{\prime}=\mathcal{M}$. Thus $\mathcal{N}$ is a direct summand of $\mathcal{M}$.

Proposition 3.10. Let $R$ be regular and $F$-finite, and let $\left(\mathcal{M}, \vartheta^{e}\right)$ be a finitely generated unit $R\left[F^{e}\right]$-module that is semisimple as a $D_{R}$-module. For some $r>0$, all $D_{R}$-isotypic components of $M$ are $R\left[F^{e r}\right]$-submodules of $\mathcal{M}$.

Proof. Let $\mathcal{M}=\mathcal{M}_{1} \oplus \ldots \oplus \mathcal{M}_{s}$ be the decomposition of $\mathcal{M}$ into its isotypic components as a $D_{R}$-module, i.e., $\mathcal{M}_{i} \cong \mathcal{N}_{i}^{\oplus n_{i}}$ for distinct, simple $D_{R^{-}}$modules $\mathcal{N}_{i}$.

By Frobenius descent, $F^{e *} \mathcal{N}_{i}$ are again distinct (pairwise nonisomorphic) simple $D_{R}$-modules. Since $F^{e *}$ commutes with finite direct sums, it follows that $F^{e^{*}} \mathcal{M}_{1} \oplus$ $\ldots \oplus F^{e *} \mathcal{M}_{s}$ is the isotypic decomposition of $F^{e *} \mathcal{M}$. Since $\vartheta^{e}$ is a $D_{R}$-module isomorphism, it maps an isotypic component $F^{e *} \mathcal{M}_{i}$ isomorphically onto another isotypic component $\mathcal{M}_{\sigma(i)}$ for some permutation $\sigma$ of the index set $\{1, \ldots, s\}$. For $r$, the order of $\sigma$ (i.e., $\sigma^{r}=$ id), it follows that $\vartheta^{e r}$ is an isomorphism of $F^{e r *} \mathcal{M}_{i}$ onto $\mathcal{M}_{i}$ for all $i$. Thus, the $D_{R}$-isotypic decomposition of $\mathcal{M}$ is, in fact, a decomposition of $\mathcal{M}$ as a unit $R\left[F^{e r}\right]$-module.

Corollary 3.11. Let $R$ be regular and $F$-finite, and let $\mathcal{M}$ be a finitely generated unit $R[F]$-module that is semisimple as a $D_{R}$-module. Each $D_{R}$-isotypic component of $\mathcal{M}$ is an $R[F]$-submodule of $\mathcal{M}$.

In particular, a simple, finitely generated unit $R[F]-$ module $\mathcal{M}$ is $D_{R}$-isotypic, i.e., as a $D_{R}$-module, $\mathcal{M} \cong \mathcal{N}^{\oplus n}$ for some simple $D_{R}$-module $\mathcal{N}$.

As a consequence, we get that a simple $D_{R}$-submodule $\mathcal{N}$ of a unit $R[F]$-module $\mathcal{M}$ carries also a unit $R[F]$-structure, although the inclusion $\mathcal{N} \subseteq \mathcal{M}$ is only $R[F]$ linear if $F_{\mathcal{M}}^{e}(\mathcal{N}) \subseteq \mathcal{N}$, which is in general not the case. 
Proposition 3.12. Let $R$ be regular and $F$-finite. Let $\mathcal{M}$ be a finitely generated unit $R[F]$-module that has finite unit $R[F]$-module length. Let $\mathcal{N}$ be a $D_{R^{-}}$subquotient of $\mathcal{M}$. Then $\mathcal{N}$ carries a unit $R[F]$-module structure.

Proof. Since the category of unit $R[F]$-modules is closed under extensions, we can reduce, by induction on the $D_{R}$-module length of $\mathcal{N}$ (which is finite by Theorem 3.4), to the case of $\mathcal{N}$ being $D_{R}$-simple.

Using that $\mathcal{M}$ has finite length as a unit $R[F]-$ module and as a $D_{R}$-module, one reduces to the case that $\mathcal{M}$ itself is simple as a unit $R[F]$-module. Then, by Corollary 3.11, $\mathcal{M}$ is semisimple as a $D_{R}$-module and $\mathcal{N}$-isotypic, i.e., as a $D_{R^{-}}$ module, $\mathcal{M} \cong \mathcal{N}^{\oplus n}$. Let $\vartheta^{e}$ be the structural morphism of $\mathcal{M}$. Let $\pi_{i}: \mathcal{M} \longrightarrow \mathcal{N}$ be the projection onto the $i$ th direct summand. The composition

$$
F^{e *} \mathcal{N} \subseteq F^{e *} \mathcal{M} \stackrel{\vartheta^{e}}{\longrightarrow} \mathcal{M} \stackrel{\pi_{i}}{\longrightarrow} \mathcal{N}
$$

is nonzero for at least one index $i$, since $\vartheta^{e}$ is an isomorphism. As a $D_{R}$-linear map between simple modules, it must be an isomorphism. Thus $F^{{ }^{*}} \mathcal{N} \cong \mathcal{N}$, and $\mathcal{N}$ is a unit $R[F]$-module.

Note that the constructed unit $R[F]$-structure on $\mathcal{N}$ is by no means unique 3 neither are the maps which express $\mathcal{N}$ as a sub-quotient of $\mathcal{M}$ maps of $R[F]-$ modules.

3.4. Connection to vector spaces with Frobenius action. By definition, a $D_{R}\left[F^{e}\right]$-module structure on $\mathcal{M}$ is a $D_{R}$-linear map $\vartheta^{e}: F^{e *} \mathcal{M} \longrightarrow \mathcal{M}$, where $F^{e *} \mathcal{M}$ carries its natural $D_{R}$-structure given by Frobenius descent. Thus, the set of $D_{R}\left[F^{e}\right]$-module structures on $\mathcal{M}$ can be identified with $\operatorname{Hom}_{D_{R}}\left(F^{e *} \mathcal{M}, \mathcal{M}\right)$. If $\mathcal{M}$ admits a unit $D_{R}\left[F^{e}\right]$-structure $\vartheta^{e}: F^{e *} \mathcal{M} \cong \mathcal{M}$, this induces an isomorphism $\operatorname{Hom}_{D_{R}}\left(F^{e *} \mathcal{M}, \mathcal{M}\right) \cong \operatorname{End}_{D_{R}}(\mathcal{M})$.

Lemma 3.13. Let $\left(\mathcal{M}, \vartheta^{e}\right)$ be a unit $D_{R}\left[F^{e}\right]$-module. Every $D_{R}\left[F^{e}\right]$-structure $\vartheta^{\prime e}$ on $\mathcal{M}$ can be written as $\vartheta^{\prime e}=\varphi \circ \vartheta^{e}$ for some unique $\varphi \in \operatorname{End}_{D_{R}}(\mathcal{M})$.

Proof. The map $\vartheta^{\prime e} \mapsto \vartheta^{\prime e} \circ\left(\vartheta^{e}\right)^{-1}$ is inverse to the map $\varphi \mapsto \varphi \circ \vartheta^{e}$.

Furthermore, $\vartheta^{\prime}$ defines a unit $D_{R}[F]$-structure if and only if the associated $\varphi=\vartheta^{\prime e} \circ\left(\vartheta^{e}\right)^{-1}$ in $\operatorname{End}_{D_{R}}(\mathcal{M})$ is a $D_{R}$-module automorphism of $\mathcal{M}$. Thus the unit $D_{R}\left[F^{e}\right]$-structures are in one-to-one correspondence with $\operatorname{Aut}_{D_{R}}(\mathcal{M})$.

Also note that the Frobenius actions on $\mathcal{M}$ corresponding to $\vartheta^{e}$ and $\vartheta^{\prime e}$ are related by $F^{\prime e}=\varphi \circ F^{e}$.

Now assume that $\mathcal{M}$ is $D_{R}$-isotypic. Then $\mathcal{M} \cong V \otimes_{k} \mathcal{N}$ for some finitedimensional $k$-vector-space $V$ and a simple $D_{R}$-module $\mathcal{N}$. In this way of writing things a differential operator $\delta$ acts as $\delta(v \otimes n)=v \otimes \delta(n)$. This is well defined since $k$ is perfect.

In order to study the Frobenius actions on $\mathcal{M}=V \otimes_{k} \mathcal{N}$ via the Frobenius actions on $V$, we assume that $\operatorname{End}_{D_{R}}(\mathcal{N})=k$. This allows us to identify the $D_{R}$-module endomorphisms of $\mathcal{M}$ with the $k$-linear endomorphisms of $V$.

\footnotetext{
${ }^{3}$ In general, the unit $D_{R}\left[F^{e}\right]$-structures of a $D_{R}$-simple module $\mathcal{N}$ correspond to $\operatorname{End}_{D_{R}}(\mathcal{N})$, which is expected to be a rather controllable set if $\mathcal{N}$ is $D_{R}$-simple, $c f$. 3.4.1
} 
3.4.1. Discussion of the assumption $\operatorname{End}_{D_{R}}(\mathcal{N})=k$. In characteristic zero, under the assumption that $R$ is a regular ring, essentially of finite type over $k$, Quillen's lemma [18] shows that the $D_{R}$-module endomorphism set of a simple $D_{R}$-module is algebraic over $k$. Thus, if $k$ is algebraically closed, $\operatorname{End}_{D_{R}}(\mathcal{N})=k$. Quillen's proof exploits the fact that $D_{R}$, in characteristic zero, is a finitely generated algebra over $k$. This fails in finite characteristic. Nevertheless, one has the following result due to Dixmier [7]; for convenience we also recall the proof.

Lemma 3.14. Let $R$ be a $k$-algebra such that the cardinality of $k$ is strictly bigger than the cardinality of a $k$-basis of $R$. If $\mathcal{N}$ is a simple $D_{R^{-}}$module, then $\operatorname{End}_{D_{R}}(\mathcal{N})$ is algebraic over $k$.

Proof. Let $\kappa$ be the cardinality of the $k$-basis of $R$ and $\kappa^{\prime}$ the strictly bigger cardinality of $k$. Since $\mathcal{N}$ is simple, we have $D_{R} n=\mathcal{N}$ for some (every nonzero) $n \in \mathcal{N}$. Thus every $\varphi \in \operatorname{End}_{D_{R}}(\mathcal{N})$ is determined by its value on $n$. Since $D_{R}$ is at most $\kappa$-dimensional over $k$, so is $\mathcal{N}=D_{R} n$, and thus $\operatorname{End}_{D_{R}}(\mathcal{N})$ is also at most $\kappa$-dimensional over $k$. Therefore, for any fixed $\varphi \in \operatorname{End}_{D_{R}}(\mathcal{N})$ (say $\varphi \notin k$ ), the set $\left\{(\varphi+\lambda)^{-1} \mid \lambda \in k\right\}$ has cardinality $\kappa^{\prime}>\kappa$, thus must be linearly dependent (we use that $\operatorname{End}_{D_{R}}(\mathcal{N})$ is a division ring by Schur's lemma). A relation of linear dependence among some finitely many $\left(\varphi+\lambda_{i}\right)^{-1}$ gives, after clearing denominators, an algebraic relation for $\varphi$. Clearing denominators works just as in the commutative case, since all $\left(\varphi+\lambda_{i}\right)^{-1}$ commute with each other.

It is an interesting open problem whether an analog of Quillen's lemma holds in finite characteristic.

For the rest of this section we assume that $\operatorname{End}_{D_{R}}(N)=k$. In the next section we will be able to put ourselves in a situation of Lemma 3.14 so that we are able to apply the following results.

Lemma 3.15. Let $\mathcal{M} \cong V \otimes_{k} \mathcal{N}$ for a simple $D_{R}$-module $\mathcal{N}$ such that $\operatorname{End}_{D_{R}}(\mathcal{N})=$ $k$. Then $\operatorname{End}_{D_{R}}(\mathcal{M}) \cong \operatorname{End}_{k}(V)$.

Tensoring with $\mathcal{N} \otimes$ _ gives a one-to-one correspondence between the $k$-vector subspaces of $V$ and the $\bar{D}_{R}$-submodules of $\mathcal{N} \otimes V \cong \mathcal{M}$.

Proof. After the choice of a basis for $V$, the $\operatorname{ring} \operatorname{End}_{D_{R}}(\mathcal{M})$ is the matrix algebra over $\operatorname{End}_{D_{R}}(\mathcal{N})=k$ of size $\operatorname{dim}_{k} V$. This identifies $\operatorname{End}_{D_{R}}(\mathcal{M})$ with $\operatorname{End}_{k}(V)$. Given a $\varphi_{k} \in \operatorname{End}_{k}(V)$, the corresponding map in $\operatorname{End}_{D_{R}}(\mathcal{M})$ is $\operatorname{id}_{\mathcal{N}} \otimes \varphi_{k}$.

Let $\mathcal{M}^{\prime}$ be a $D_{R^{-}}$submodule of $\mathcal{M}$. Since $\mathcal{M}$ is semisimple, we find a $D_{R^{-}}$ submodule $\mathcal{M}^{\prime \prime}$ such that $\mathcal{M}^{\prime} \oplus \mathcal{M}^{\prime \prime} \cong \mathcal{M}$. Then $\mathcal{M}^{\prime}$ is the kernel of the endomorphism $\varphi: \mathcal{M} \stackrel{\pi}{\longrightarrow} \mathcal{M}^{\prime \prime} \subseteq \mathcal{M}$, where $\pi$ is the projection onto the direct summand $\mathcal{M}^{\prime \prime}$. Thus, by the first part, $\varphi=\operatorname{id}_{\mathcal{N}} \otimes \varphi_{k}$ for some $\varphi_{k}$ in $\operatorname{End}_{k}(V)$. Then clearly $\mathcal{M}^{\prime}=V^{\prime} \otimes \mathcal{N}$, with $V^{\prime}=\operatorname{ker} \varphi_{k}$.

I want to extend the last lemma so that it incorporates Frobenius operations. Then it is possible to reduce questions about unit $R[F]$-submodules of $M$ (e.g., are there any nontrivial ones?) to the equivalent questions about $k[F]$-subspaces of $V$. The main observation is:

Proposition 3.16. Let $\mathcal{M}=V \otimes_{k} \mathcal{N}$ be as in the last lemma. Assume that $\mathcal{M}$ is a unit $D_{R}\left[F^{e}\right]$-module with Frobenius action $F_{\mathcal{M}}^{e}$. Then there is a Frobenius action $F_{V}^{e}$ on $V$ such that the $D_{R}\left[F^{e}\right]$-submodules of $\mathcal{M}$ are in one-to-one correspondence with the $k\left[F^{e}\right]$-submodules of $V$. 
Proof. First observe that by Proposition 3.12, $\mathcal{N}$ carries some (not necessarily unique) unit $D_{R}\left[F^{e}\right]$-module structure. Denote the corresponding Frobenius action by $F_{\mathcal{N}}^{e}$. The choice of a basis of $V$ equips $V$ with a unit $k\left[F^{e}\right]$-structure by letting $F^{e}$ act as the identity on the basis and extending $p^{e}$ linearly. This Frobenius action we denote by $F_{V}^{\prime e}$. Then $F_{\mathcal{M}}^{\prime e} \stackrel{\text { def }}{=} F_{\mathcal{N}}^{e} \otimes F_{V}^{\prime e}$ defines a unit $D_{R}\left[F^{e}\right]$-structure. The corresponding Frobenius structure on $\mathcal{M}$ is given by

$$
\left(R^{e} \otimes_{R} \mathcal{N}\right) \otimes_{k} V \longrightarrow \mathcal{N}^{e} \otimes_{k} V \cong \mathcal{N} \otimes_{k}\left(k^{e} \otimes_{k} V\right) \longrightarrow \mathcal{N} \otimes_{k} V
$$

where the first map is the unit $R\left[F^{e}\right]$-structure on $\mathcal{N}$ and the last is the unit $k\left[F^{e}\right]$-structure on $V$. Since these both are isomorphisms, so is the composition.

By Lemma 3.13, we can express $F_{\mathcal{M}}^{e}=\varphi \circ{F^{\prime}}_{\mathcal{M}}^{e}$ for some $\varphi \in \operatorname{Aut}_{D_{R}}(\mathcal{M})$. By Lemma 3.15 we can write $\varphi=\operatorname{id}_{\mathcal{N}} \otimes \varphi_{V}$ for some $k$-vector-space automorphism $\varphi_{V}$ of $V$. Denoting the corresponding unit $k\left[F^{e}\right]$-structure on $V$ by $F_{V}^{e} \stackrel{\text { def }}{=} \varphi_{V} \circ F_{V}^{\prime e}$, one easily verifies that $F_{\mathcal{M}}^{e}=F_{\mathcal{N}}^{e} \otimes F_{V}^{e}$. Indeed,

$$
F_{\mathcal{N}}^{e} \otimes F_{V}^{e}=F_{\mathcal{N}}^{e} \otimes\left(\varphi_{V} \circ F_{V}^{\prime e}\right)=\varphi \circ\left(F_{\mathcal{N}}^{e} \otimes F_{V}^{\prime e}\right)=\varphi \circ F_{\mathcal{M}}^{e}=F_{\mathcal{M}}^{e}
$$

With $F_{V}^{e}$ we have constructed the desired Frobenius action on $V$. Since $F_{\mathcal{M}}^{e}=$ $F_{\mathcal{N}}^{e} \otimes F_{V}^{e}$, we have $F_{\mathcal{M}}^{e}\left(\mathcal{N} \otimes V^{\prime}\right)=\mathcal{N} \otimes F_{V}^{e}\left(V^{\prime}\right)$. Therefore, a $D_{R}$-submodule of the form $\mathcal{N} \otimes V^{\prime}$ of $\mathcal{M}$ is stable under $F_{\mathcal{M}}^{e}$ if and only if the subspace $V^{\prime}$ of $V$ is stable under $F_{V}^{e}$. By Lemma 3.15 every $D_{R^{-}}$submodule of $\mathcal{M}$ is of this form.

Note that by Proposition 3.6 the $D_{R}[F]$-submodules of $\mathcal{M}$ are exactly the unit $R[F]$-submodules. One gets the following corollary.

Corollary 3.17. With the notation as in the last proposition, the unit $R\left[F^{e}\right]-$ submodules of $\left(\mathcal{M}, F_{\mathcal{M}}^{e}\right)$ are in one-to-one correspondence with the $k\left[F^{e}\right]$-submodules of $\left(V, F_{V}^{e}\right)$.

In particular, $\mathcal{M}$ is a simple unit $R[F]-$ module if and only if $V$ is a simple $k[F]-$ module.

\section{LENGTH OF UNIT $R[F]$-MODUleS}

The length of an object $\mathcal{M}$ in an abelian category (the length of the longest chain of proper inclusions of objects in the category starting with zero and ending with $\mathcal{M}$ ) is denoted by small $l_{*}$ decorated by a modifier pointing out the category. For example, if $\mathcal{M}$ is a finitely generated unit $R\left[F^{e}\right]$-module, we denote

$$
\begin{aligned}
l_{u R\left[F^{e}\right]}(\mathcal{M}) & =\text { the length of the unit } R\left[F^{e}\right]-\text { module } \mathcal{M}, \\
l_{u R\left[F^{e r}\right]}(\mathcal{M}) & =\text { the length of } \mathcal{M} \text { as a unit } R\left[F^{e r}\right] \text {-module, } \\
l_{D_{R}}(\mathcal{M}) & =\text { the length of } \mathcal{M} \text { as a } D_{R^{-}} \text {module, } \\
l_{u R[F]}(\mathcal{M}) & =\text { the length of } \mathcal{M} \text { as a unit } R[F]-\text { module. }
\end{aligned}
$$

One easily concludes the following proposition.

Proposition 4.1. Let $R$ be essentially of finite type over the perfect field $k$. Let $\mathcal{M}$ be a finitely generated unit $R\left[F^{e}\right]$-module. Then there is an $r>0$ such that

$$
l_{u R\left[F^{e}\right]}(\mathcal{M}) \leq l_{u R\left[F^{e r}\right]}(\mathcal{M})=l_{u R[F]}(\mathcal{M}) \leq l_{D_{R}}(\mathcal{M}),
$$

and furthermore, all these lengths are finite. 
Proof. By the above Theorem [3.4, the length of $\mathcal{M}$ as a $D_{R}-$ module is finite. The inclusion of categories

$$
u R\left[F^{e}\right]-\bmod \subseteq u R[F]-\bmod \subseteq D_{R^{-}} \bmod
$$

shows the claimed inequality of lengths. The equality follows, since a chain of finitely many $R[F]$-modules is a chain of $R\left[F^{e r}\right]$-modules for some $r>0$.

More interesting is the case when one asks what happens after extending the perfect field $k \subseteq R$ over which $R$ is essentially of finite type. If $K$ is such an extension field of $k$, then we denote $R_{K}=K \otimes_{k} R$. Clearly, tensoring with $K \otimes_{k}-$ is a functor from $u R\left[F^{e}\right]-\bmod$ to $u R_{K}\left[F^{e}\right]-\bmod$, from $u R[F]-\bmod$ to $u R_{K}[F]-\bmod$ and from $D_{R}-\bmod$ to $D_{R_{K}}-\bmod$, preserving finite generation. Furthermore, this functor can only increase the length, since it is faithful.

Definition 4.2. Let $\mathcal{M}$ be a finitely generated unit $R\left[F^{e}\right]$-module. The geometric length of $\mathcal{M}$ is defined as the length of $K \otimes_{k} \mathcal{M}$, where $K$ is an algebraically closed field containing $k$. This notion of geometric length applies to all the lengths introduced above, and is denoted by $\bar{l}_{*}$, where $*$ is the appropriate category.

For this to make sense, one has to show that the length does not depend on the chosen algebraically closed field $K$. For the categories of finitely generated unit $R\left[F^{e}\right]$-modules and $R[F]$-modules, this is a consequence of the next proposition. The case of $D_{R}$-submodules of unit $R[F]$-modules is treated in the next subsection as a byproduct of Theorem 4.5 .

Proposition 4.3. Let $R$ be essentially of finite type over an algebraically closed field $k$, and let $K$ be an algebraically closed extension field. If $\mathcal{M}$ is a simple finitely generated unit $R\left[F^{e}\right]$-module (resp. $R[F]$-module), then $K \otimes_{k} \mathcal{M}$ is also simple as a unit $R_{K}\left[F^{e}\right]$-module (resp. $R_{K}[F]$-module).

Proof. The proof of this is a fairly standard argument using the Nullstellensatz and generic flatness 4 In order to apply these techniques, one has to work with finitely generated $R$-modules. Thus the trick consists of using the roots of the unit $R\left[F^{e}\right]$-modules in question. These are finitely generated $R$-modules.

Let $\beta: M \longrightarrow F^{e *} M$ be a root of $\mathcal{M}$. Then $\beta_{K}: M_{K}=K \otimes_{k} M \longrightarrow F_{R_{K}}^{e *} M_{K}$ is a root of $\mathcal{M}_{K}=K \otimes_{k} \mathcal{M}$. Let $\mathcal{N}$ be a nonzero unit $R\left[F^{e}\right]$-submodule of $\mathcal{M}_{K}$. Then the restriction of $\beta_{K}$ to $N_{K}=M_{K} \cap \mathcal{N}$ is a root of $\mathcal{N}$. Since both $M_{K}$ and $N_{K}$ are finitely presented, one can find free presentations

$$
R_{K}^{\oplus n^{\prime}} \longrightarrow R_{K}^{\oplus m^{\prime}} \longrightarrow M_{K} \longrightarrow 0 \quad \text { and } \quad R_{K}^{\oplus n} \longrightarrow R_{K}^{\oplus m} \longrightarrow N_{K} \longrightarrow 0
$$

\footnotetext{
${ }^{4}$ Thanks go to Brian Conrad for suggesting that these techniques could be applied here.
} 
The root morphism $\beta_{K}$ as well as the inclusion $i_{K}: N_{K} \longrightarrow M_{K}$ can be extended to a commutative diagram:

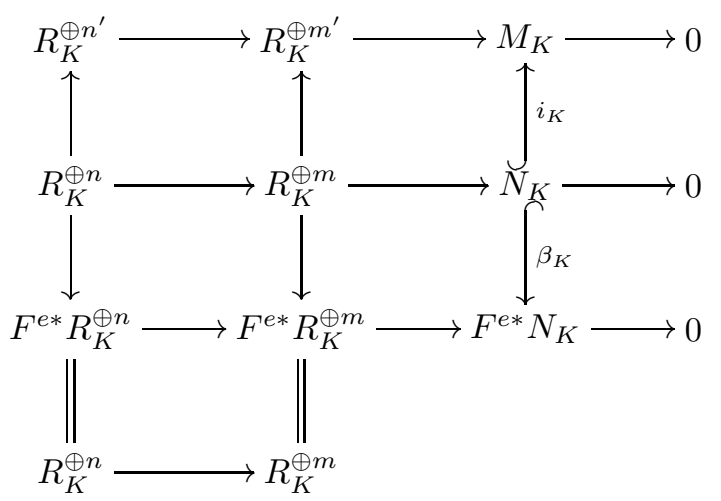

The third row is just the Frobenius functor $F_{R_{K}}^{e *}$ applied to the second row. The equal signs indicate the natural unit $R\left[F^{e}\right]$-structure on $R$. Thus, the six maps of free $R_{K}$-modules constituting the left big rectangle completely determine the root morphisms $\beta_{K}$ and the inclusion $i_{k}: N_{K} \subseteq M_{K}$. Since these are maps of finitely generated free $R_{K}$-modules, they are defined over a finitely generated $k$-algebra $A \subseteq K$ : just adjoin to $k$ all the coefficients of the matrices representing these maps. Then these maps are, in fact, maps of free $R_{A}=\left(A \otimes_{k} R\right)$-modules. We get the corresponding diagram of $R_{A}$-modules defining $M_{A}$ and $N_{A}$ as the cokernels of these maps of free modules (clearly, $M_{A} \cong A \otimes_{k} M$ ):

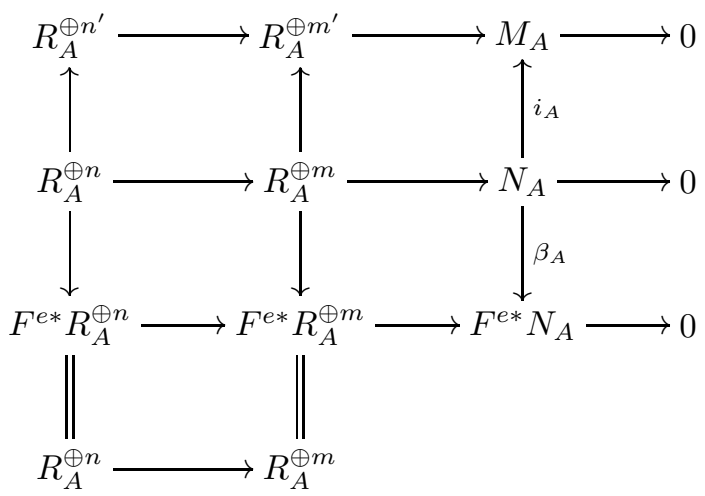

Since $F^{e *}$ is right exact, the cokernel of the third row is in fact $F_{R_{A}}^{e *} N_{A}$ as indicated. The map $\beta_{A}$ and the map $i_{A}$ are the ones induced on cokernels. By further enlarging $A$ one can assume that all the modules involved (and especially the kernels of $\beta_{A}$ and $i_{A}$ ) are free $A$-modules by generic flatness, 8], Theorem 14.4. Obviously, tensoring this diagram with $K$ over $A$, one gets back diagram (3). Since we chose $A$ such that the kernel of $\beta_{A}$ is free, it follows that this kernel must be zero, since it is zero after tensoring with $K$. Similarly, the kernel of $i_{A}$ is also zero. Thus $\beta_{A}$ and $i_{A}$ are injective. Thus we can think of $\beta$ as the root of a finitely generated unit $R_{A}\left[F^{e}\right]$-submodule $\mathcal{N}_{A}$ of $\mathcal{M}_{A}=A \otimes_{k} \mathcal{M}$.

Now, let $m$ be a maximal ideal of $A$. Then, reducing $\bmod m$, we see that $N_{k} \stackrel{\text { def }}{=} A / m \otimes_{A} N_{A}$ is a root of the unit $R\left[F^{e}\right]$-submodule $\mathcal{N}_{k} \stackrel{\text { def }}{=} A / m \otimes_{A} \mathcal{N}_{A}$ of $\mathcal{M}$ (here we used the Nullstellensatz and the algebraic closedness of $k$ to conclude 
that $A / m \otimes_{A} R_{A} \cong R$, since $A / m=k$ ). Again, by ensuring that $N_{A}$ is free over $A$, it follows that $N_{k}$ and therefore $\mathcal{N}_{k}$ is nonzero. Since $\mathcal{M}$ is simple, it follows that $\mathcal{N}_{k}=\mathcal{M}$. But this implies that $N_{k}=M$, and therefore $N_{A}=M_{A}$. Thus $N_{K}=M_{K}$, and thus $\mathcal{N}_{K}=\mathcal{M}_{K}$. This implies that $\mathcal{M}_{K}$ is a simple unit $R_{K}\left[F^{e}\right]-$ module.

The case of $\mathcal{M}$ being a simple $R[F]-$ module follows easily. $\mathcal{M}$ is a simple $R\left[F^{e}\right]_{-}$ module for infinitely many $e$. Thus $\mathcal{M}_{K}$ is a simple $R_{K}\left[F^{e}\right]$-module for such $e$, and therefore also a simple $R_{K}[F]$-module.

As a corollary of the proof of the proposition, one gets

Corollary 4.4. Let $R$ be essentially of finite type over a perfect field $k$. Let $\mathcal{M}$ be a finitely generated unit $R\left[F^{e}\right]-$ module. Then there is a finite algebraic extension field $k^{\prime}$ of $k$ such that $\bar{l}_{u R\left[F^{e}\right]}(\mathcal{M})=l_{R_{k^{\prime}}\left[F^{e}\right]}\left(\mathcal{M}_{k^{\prime}}\right)$ and similarly for the unit $R[F]-$ module length.

Proof. After tensoring with the algebraic closure $K$, one has a finite sequence of unit $R_{K}\left[F^{e}\right]$-submodules of $\mathcal{M}_{K}$ :

$$
0 \subseteq \mathcal{M}^{1} \subseteq \mathcal{M}^{2} \subseteq \ldots \subseteq \mathcal{M}^{s}=\mathcal{M}_{K}
$$

where $s$ is the geometric $R\left[F^{e}\right]$-module length of $\mathcal{M}$. Similarly as in the proof of the last proposition, i.e., turning to presentations of the roots, we see that all these $M^{i}$ 's arise as $K \otimes_{k} M_{k^{\prime}}^{i}$, where $M_{k^{\prime}}^{i}$ are unit $R_{K}\left[F^{e}\right]$-submodules of $M_{k^{\prime}}$ ( $k^{\prime}$ takes the role of $A$ in the last proof). This shows that the unit $R_{k^{\prime}}\left[F^{e}\right]$-module length of $M_{k^{\prime}}$ is equal to the geometric unit $R\left[F^{e}\right]$-module length of $\mathcal{M}$.

The case of unit $R[F]$-module length is achieved by looking at $R\left[F^{e}\right]$-module lengths for sufficiently big $e$.

4.1. Geometric $F$-length is geometric $D_{R}$-length. The fact that the geometric $D_{R}$-module length is well defined will be proved by showing that for sufficiently huge fields $K$, the $D_{R_{K}}$-module length is equal to the $R_{K}[F]$-module length. Then, if $R$ is essentially of finite type over the algebraically closed field $k$, we have the chain of inequalities

$$
\bar{l}_{u R[F]} \leq l_{D_{R}} \leq l_{D_{R_{K}}}=l_{u R_{K}[F]} .
$$

Therefore equality prevails everywhere, and the geometric $D_{R}$-module length is also well defined. The crucial step is therefore the following theorem.

Theorem 4.5. Let $k$ be algebraically closed and of strictly bigger cardinality than a $k$-basis of $R$. Then a simple finitely generated unit $R[F]$-module $\mathcal{M}$ is simple as a $D_{R}$-module.

Proof. By Corollary $3.11\left(\mathcal{M}, \vartheta_{\mathcal{M}}^{e}, F_{\mathcal{M}}^{e}\right)$ is $D_{R}$-isotypic. Hence, as a $D_{R}$-module, $\mathcal{M}$ is isomorphic to $V \otimes_{k} \mathcal{N}$ for a simple $D_{R}-\operatorname{module} \mathcal{N}$ and a finite-dimensional $k^{-}$ vector-space $V$. The cardinality assumption together with the algebraic closedness of $k$ implies (Lemma 3.14) that $\operatorname{End}_{D_{R}}(\mathcal{N})=k$. Thus we are in the situation of Proposition [3.16, and therefore obtain a unit $k\left[F^{e}\right]$-structure $F_{V}^{e}$ on $V$ such that the unit $R[F]$-submodules of $\mathcal{M}$ are in one-to-one correspondence with the $k[F]$ submodules of $V$. In particular, $\mathcal{M}$ is simple as a unit $R[F]$-module if and only if $V$ has no nontrivial $F_{V}^{e}$-stable submodules. The following Proposition 4.6] of Dieudonné then shows that $V$ is one-dimensional. Therefore $\mathcal{M} \cong \mathcal{N}$, and $\mathcal{M}$ is $D_{R}$-simple. 
The last technical aid is Dieudonné [6], Proposition 3, page 233, the statement of which we recall.

Proposition 4.6. Let $k$ be an algebraically closed field and $V$ a finite-dimensional $k\left[F^{e}\right]$-vector-space. If the Frobenius $F^{e}$ acts injectively on $V$, then $V$ has a basis consisting of $F^{e}$-fixed elements of $V$.

As a corollary of the last theorem we get the equality of geometric $R[F]$-module length and $D_{R}$-module length.

Corollary 4.7. Let $R$ be regular, essentially of finite type over a field $k$, and let $\mathcal{M}$ be a finitely generated unit $R[F]$-module. Then the geometric $D_{R}$-module length of $\mathcal{M}$ is well defined and equal to the geometric unit $R[F]-$ module length of $\mathcal{M}$.

Proof. Since the geometric unit $R[F]$-module length is well defined (Proposition 4.3),

$$
\bar{l}_{u R[F]}(\mathcal{M})=l_{u R_{K}[F]}\left(\mathcal{M}_{K}\right)
$$

with $K$ any algebraically closed extension field of $k$. Given a maximal filtration of $\mathcal{M}_{K}$ as a unit $R_{K}[F]-$ module,

$$
0 \subseteq \mathcal{M}^{1} \subseteq \ldots \subseteq \mathcal{M}^{l}=\mathcal{M}_{K}
$$

with simple unit $R_{K}[F]$-module quotients if $K$ is sufficiently big (uncountable), the last theorem shows that all the quotients are $D_{R_{K}}$-simple. Therefore the geometric $D_{R}$-module length of $\mathcal{M}$ is equal to $l=\bar{l}_{u R[F]}$, and thus it too is well defined (independent of $K$ ).

\section{EXAMPLES}

An example of a simple $R[F]$-module that is not simple as a $D_{R}$-module is given. This example is constructed as a free $R$-module $\mathcal{M}$ of rank 2 . Let the action of the Frobenius $F^{e}$ with respect to some basis $\mathcal{A}=\left(e_{1}, e_{2}\right)$ be represented by the matrix

$$
A=\left(\begin{array}{ll}
0 & 1 \\
1 & x
\end{array}\right)
$$

for some element $x \in R$, i.e., for $v=v_{1} e_{1}+v_{2} e_{2}$ the action of $F^{e}$ is given by

$$
v=\left(\begin{array}{c}
v_{1} \\
v_{2}
\end{array}\right) \mapsto A\left(\begin{array}{c}
v_{1}^{q} \\
v_{2}^{q}
\end{array}\right) .
$$

The choice of basis $\mathcal{A}$ also induces a natural $D_{R}$-structure on $\mathcal{M} \cong R \oplus R$ by acting componentwise on the direct summands. Unless otherwise specified, this is the $D_{R}$-module structure on $\mathcal{M}$ we have in mind.

The matrix $A_{r}$ representing the $r$ th power of this Frobenius action $F^{e}$ with respect to this basis is given by

$$
A_{r}=A A^{[q]} \cdots A^{\left[q^{r-1}\right]}
$$

where the square brackets $[q]$ raise each coefficient of the matrix to its $q$ th power. Equivalently, $A_{r}$ can be described inductively by the equation $A_{r}=A_{r-1} A^{\left[q^{r-1}\right]}$, which translates into an inductive formula for the coefficients of $A_{r}$. One has

$$
A_{r}=\left(\begin{array}{cc}
a_{r-2}^{q} & a_{r-1}^{q} \\
a_{r-1} & a_{r}
\end{array}\right),
$$


where $a_{r}=a_{r-2}+a_{r-1} x^{q^{r-1}}$ with $a_{-1}=0$ and $a_{0}=1$. So, for example, this formula computes as

$$
A_{1}=\left(\begin{array}{ll}
0 & 1 \\
1 & x
\end{array}\right) \text { and } A_{2}=\left(\begin{array}{cc}
1 & x^{q} \\
x & x^{q+1}+1
\end{array}\right)
$$

which can be easily verified by hand. We prove these assertions by induction:

$$
\begin{aligned}
A_{r}=A_{r-1} A^{\left[q^{r-1}\right]} & =\left(\begin{array}{cc}
a_{r-2}^{q} & a_{i-3}^{q}+a_{r-2}^{q} x^{q^{r-1}} \\
a_{i-1} & a_{a-2}+a_{i-1} x^{q^{r-1}}
\end{array}\right) \\
& =\left(\begin{array}{cc}
a_{r-2}^{q} & \left(a_{r-3}+a_{r-2} x^{q^{r-2}}\right)^{q} \\
a_{r-1} & a_{r}
\end{array}\right)=\left(\begin{array}{cc}
a_{2-1}^{q} & a_{r-1}^{q} \\
a_{r-1} & a_{r}
\end{array}\right),
\end{aligned}
$$

where the base case is by the initial condition of the recursion for $a_{r}$. Furthermore, we note that, thinking of $a_{r}$ as polynomials in $x$, the degree in $x$ of $a_{r}$ is $\operatorname{deg} a_{r}=$ $1+q+q^{2}+\ldots+q^{r-1}$. Again an induction argument shows this nicely:

$$
\begin{aligned}
\operatorname{deg} a_{r} & =\max \left\{\operatorname{deg} a_{r-2}, \operatorname{deg} a_{r-1}+q^{r-1}\right\} \\
& =\max \left\{1+q+\ldots q^{r-3}, 1+q+\ldots+q^{r-2}+q^{r-1}\right\} \\
& =1+q+\ldots+q^{r-2}+q^{r-1},
\end{aligned}
$$

and for the start of the induction we just recall that $a_{-1}=0$ and $a_{0}=1$. This setup is used as the basis for the following examples.

5.0.1. A simple $R\left[F^{1}\right]$-module that is not $D_{R}$-simple. Let $R=\mathbb{F}_{3}$ and $x=1$. Since the Frobenius $F$ is the identity on $\mathbb{F}_{3}$, Frobenius actions are just linear maps. The linear map represented by

$$
A=\left(\begin{array}{ll}
0 & 1 \\
1 & 1
\end{array}\right)
$$

is not diagonalizable, since its characteristic polynomial $P_{A}(t)=t(t-1)+1$ is irreducible over $\mathbb{F}_{3}$. Thus $\mathcal{M}$ is a simple $\mathbb{F}_{3}\left[F^{1}\right]$-module. Since $\mathbb{F}_{3}$ is perfect, we get $D_{\mathbb{F}_{3}}=\mathbb{F}_{3}$, and thus $\mathcal{M}$ is not simple as a $D_{\mathbb{F}_{3}}$-module, since it is a free $\mathbb{F}_{3}$-module

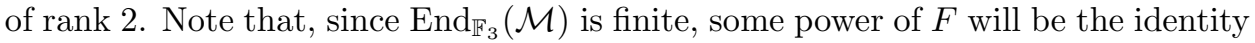
on $\mathcal{M}$. An easy calculation shows that $F^{4}=-\mathrm{id}_{M}$, and therefore $\mathcal{M}$ is not simple as a $\mathbb{F}_{3}\left[F^{4}\right]$-module.

On the other hand, after adjoining a root $\alpha$ of the polynomial $P_{A}=t(t-1)+1$ to $\mathbb{F}_{3}$, we get the extension field $k=\mathbb{F}_{3}(\alpha)$. Clearly, $k \otimes_{\mathbb{F}_{3}} \mathcal{M}$ is no longer simple as a $k\left[F^{1}\right]$-module.

5.0.2. A $D_{R}$-submodule that is not an $R[F]$-submodule. Now let $R$ be a ring containing an infinite perfect field $k$ with $x \in k$ transcendental over the prime field $\mathbb{F}_{p}$. With this $x$ the matrix $A$, in fact, represents a $D_{R}$-linear map of $\mathcal{M}$ (this is because differential operators $D_{R}$ are linear over any perfect subring of $R$ ). Since $a_{r}$ is a nonzero polynomial in $x$ with coefficients in $\mathbb{F}_{p}$, and since $x$ is transcendental over the prime field, $a_{r}$ is a nonzero element of $k$. This implies that, for example, $R e_{1}$ is not stable under any power of $F^{e}$, for this would be equivalent to the matrix $A_{r}$ having a zero entry in the bottom left corner. But this entry is $a_{r-1}$, which we just argued is nonzero. Thus $R e_{1}$ is a (simple) $D_{R}$-submodule that is not an $R[F]$-submodule of $M$. 
5.1. A simple unit $R[F]$-module that is not $D_{R}$-simple. Now we come to the main example of a simple $R[F]$-module that is not $D_{R^{-}}$simple. This will show that the unit $R[F]$-module and $D_{R}$-module lengths of a unit $R[F]$-module can be different. It provides a counterexample to Lyubeznik's Remark 5.6a in [15], where he speculates that simple $D_{R}$-submodules of simple unit $R[F]$-modules are in fact $R[F]$-submodules.

With $A$ and $\mathcal{M}$ as before, let $R=k(x)^{1 / p^{\infty}}$ be the perfect closure of $k(x)$, where $x$ is a new variable and $k$ is perfect. As before we define the Frobenius action $F^{e}$ on $\mathcal{M}$ as being represented by $A$, i.e., given by application of

$$
F^{e}\left(\_\right)=\left(\begin{array}{ll}
0 & 1 \\
1 & x
\end{array}\right)\left(乙^{\left[p^{e}\right]}\right.
$$

with respect to the basis $\mathcal{A}$. Since $R$ is perfect, $R=D_{R}$, and consequently this (and every) unit $R[F]$-structure is compatible with the $D_{R}$-structure.

To show that $\left(\mathcal{M}, F^{e}\right)$ is simple as a unit $R[F]-$ module, we show that $M$ is a simple unit $R\left[F^{e r}\right]$-module for all $r$. We proceed in 3 steps:

SteP 1. With respect to the basis $\mathcal{A}$ the action $F^{e r}$ is represented by the matrix $A_{r}$. We change the basis appropriately to $\mathcal{B}=\left(f_{1}, f_{2}\right)$ such that the representing matrix $B_{r}$ of $F^{e r}$ with respect to the basis $\mathcal{B}$ is "nice"; by this we mean that

$$
B_{r}=\left(\begin{array}{cc}
0 & s_{r} \\
1 & t_{r}
\end{array}\right)
$$

for some $s_{r}, t_{r} \in \mathbb{F}_{p}[x]$.

SteP 2. Assuming that there is a $v \in M$ such that $F^{e r}(v)=\lambda v$ yields a monic algebraic equation which gives an algebraic equation for $x$.

SteP 3. By transcendence of $x$ this equation must be zero, and we discriminate two cases to arrive at a contradiction. One is treated by a degree argument, the other by differentiation.

Let us begin with Step 1: The basis that will lead to the matrix $B_{r}$ of the desired shape is $f_{1}=e_{1}$ and $f_{2}=a_{r-2}^{q} e_{1}+a_{r-1} e_{2}$. Thus the matrix responsible for the base change from $\mathcal{A}$ to $\mathcal{B}$ is

$$
C_{r} \stackrel{\text { def }}{=}\left(\begin{array}{ll}
1 & a_{r-2}^{q} \\
0 & a_{r-1}
\end{array}\right)
$$

With respect to the new basis $\mathcal{B}$ the Frobenius action $F^{e r}$ is represented by the matrix $B_{r}=C_{r}^{-1} A_{r} C_{r}^{\left[q^{r}\right]}$. This can be checked by hand; a more thorough discussion of Frobenius actions on free $R$-modules under change of basis can be found in [3]. 
To determine $s_{r}$ and $t_{r}$ we explicitly calculate $B_{r}$ :

$$
\begin{aligned}
B_{r} & =C_{r}^{-1} A_{r} C_{r}^{\left[q^{r}\right]}=C_{r}^{-1}\left(\begin{array}{cc}
a_{r-2}^{q} & a_{r-1}^{q} \\
a_{r-1} & a_{r}
\end{array}\right)\left(\begin{array}{cc}
1 & a_{r-2}^{q^{r}+q} \\
0 & a_{r-1}^{q^{r}}
\end{array}\right) \\
& =\frac{1}{a_{r-1}}\left(\begin{array}{cc}
a_{r-1} & -a_{r-2}^{q} \\
0 & 1
\end{array}\right)\left(\begin{array}{cc}
a_{r-2}^{q} & a_{r-2}^{q} a_{r-2}^{q^{r}+q}+a_{r-1}^{q} a_{r-1}^{q^{r}} \\
a_{r-1} & a_{r-1} a_{r-2}^{q^{2}+q}+a_{r} a_{r-1}^{q^{r}}
\end{array}\right) \\
& =\left(\begin{array}{cc}
0 & -a_{r-1}^{q^{r}-1} \operatorname{det} A_{r} \\
1 & a_{r-2}^{q^{r}+q}+a_{r} a_{r-1}^{q^{r}-1}
\end{array}\right) \\
& =\left(\begin{array}{cc}
0 & (-1)^{r-1} a_{r-1}^{q^{r}-1} \\
1 & a_{r-2}^{q^{r}+q}+a_{r} a_{r-1}^{q^{r}-1}
\end{array}\right) .
\end{aligned}
$$

Besides index juggling skills, one only needs the equation $\operatorname{det} A_{r}=(-1)^{r}$, which follows from the recursive definition of $A_{r}$ and the fact that $\operatorname{det} A=-1$. We can read off the desired expressions for $s_{r}$ and $t_{r}$ :

$$
s_{r}=(-1)^{r-1} a_{r-1}^{q^{r}-1} \text { and } t_{r}=a_{r-2}^{q^{r}+q}+a_{r} a_{r-1}^{q^{r}-1} .
$$

Note that both are in $\mathbb{F}_{p}[x]$, since $a_{i} \in \mathbb{F}_{p}[x]$.

We now work over this new basis $\mathcal{B}$ and start with Step 2. Assume we have $v=(\alpha, 1)^{t}$ such that $F^{r}(v)=\lambda v$. One reduces from a general $v=(\alpha, \beta)^{t}$ to this case by dividing by $\beta ; 5.0 .2$ ensures that $\beta$ is not zero. Considering $\lambda v=B_{r} v^{\left[q^{r}\right]}$, this yields two equations:

$$
\begin{aligned}
s_{r} & =\lambda \alpha, \\
\alpha^{q^{r}}+t_{r} & =\lambda .
\end{aligned}
$$

Substituting the latter in the former, we get a monic algebraic equation for $\alpha$ with coefficients in $k[x]$ :

$$
\alpha^{q^{r}+1}+t_{r} \alpha-s_{r}=0 .
$$

Since $k[x]^{1 / p^{\infty}}$ is integrally closed in $R$, we conclude that $\alpha \in k[x]^{1 / p^{\infty}}$. Choose $t$ minimal such that $\alpha \in k\left[x^{1 / p^{t}}\right]$. Then $\beta(x)=\alpha^{p^{t}}$ is in $k[x]$, and not a $p$ th power unless $t=0$. Taking the $p^{t}$ th power of the last equation, we get

$$
\beta^{q^{r}+1}+t_{r}^{p^{t}} \beta-s_{r}^{p^{t}}=0,
$$

which is an algebraic relation for $x$ with coefficients in $k$. Thus it is constant zero by transcendence of $x$.

For Step 3 we distinguish the following two cases:

$t>0$ : Differentiating (6) with respect to $x$, we get

$$
\beta^{q^{r}} \frac{\partial}{\partial x} \beta+t_{r}^{p^{t}} \frac{\partial}{\partial x} \beta=0 .
$$

Since we chose $\beta$ not to be a $p$ th power, its derivative is nonzero. Thus we can divide the above by $\frac{\partial}{\partial x} \beta$ and get $\beta^{q^{r}}=-t_{r}^{p^{t}}$. Substituting this back into (6), we get $s_{r}^{p^{t}}=0$. But this is a contradiction, since $s_{r}= \pm a_{r-1}^{q^{r}-1} \neq 0$. 
$t=0$ : For this we have to determine the degrees of the terms in Equation ([6). As observed earlier, $\operatorname{deg}\left(a_{r}\right)=1+q+\ldots+q^{r-1}$. Therefore,

$$
\begin{aligned}
\operatorname{deg} s_{r} & =\left(q^{r}-1\right) \operatorname{deg}\left(a_{r-1}\right) \\
& =-1-q-\ldots-q^{r-2}+q^{r}+\ldots+q^{2 r-2}, \\
\operatorname{deg} t_{r} & \leq \max \left\{\operatorname{deg}\left(a_{r-2}^{q^{r}+q}\right), \operatorname{deg}\left(a_{i} a_{r-1}^{q^{r}-1}\right)\right\} \\
& =q^{r-1}+\ldots+q^{2 r-2} .
\end{aligned}
$$

In fact, equality prevails in the last inequality, since the two entries in the max are different (the second is always bigger). To be precise,

$$
\begin{aligned}
\operatorname{deg}\left(a_{r-2}^{q^{r}+q}\right)= & \left(q^{r}+q\right)\left(1+\ldots+q^{r-3}\right) \\
= & q+\ldots+q^{r-2}+q^{r}+\ldots+q^{2 r-3}, \\
\operatorname{deg}\left(a_{i} a_{r-1}^{q^{r}-1}\right)= & 1+q+\ldots+q^{r-1}-1 \\
& \quad-\ldots-q^{r-2}+q^{r}+\ldots+q^{2 r-2} \\
= & q^{r-1}+\ldots+q^{2 r-2} .
\end{aligned}
$$

Since $q^{r-1}>1+q+\ldots+q^{r-2}$, we see that the second line is in fact strictly bigger than the first. Thus the degree of $s_{r}$ is strictly smaller than the degree of $t_{r}$, and therefore the first two terms of (6) must have the same degree. If we denote the degree of $\beta$ by $n$, we get

$$
\left(q^{r}+1\right) n=\operatorname{deg}\left(t_{r}\right)+n,
$$

and after dividing by $q^{r-1}$ this simplifies to

$$
1=q n-q-q^{2}-\ldots-q^{r-1} .
$$

The right side is divisible by $q$, but the left side certainly is not. This is a contradiction.

This finishes the proof that $\mathcal{M}$ is a simple $R\left[F^{e r}\right]$-module for all $r>0$. Thus $\mathcal{M}$ is a simple $R[F]$-module, but $M$ is not simple as a $D_{R}$-module since every one-dimensional $R$-subspace is a nontrivial $D_{R}$-submodule.

Let $R^{\prime}=R(\alpha)$ be the field one obtains by adjoining to $R$ a root $\alpha$ of the polynomial $P(t)=t^{p^{2}}+x t^{p}-t$. Then

$$
F\left(\left(\begin{array}{c}
\alpha^{p} \\
\alpha
\end{array}\right)\right)=\left(\begin{array}{ll}
0 & 1 \\
1 & x
\end{array}\right)\left(\begin{array}{c}
\alpha^{p^{2}} \\
\alpha^{p}
\end{array}\right)=\left(\begin{array}{c}
\alpha^{p} \\
\alpha^{p^{2}}+x \alpha^{p}
\end{array}\right)=\left(\begin{array}{c}
\alpha^{p} \\
\alpha
\end{array}\right),
$$

which shows that the element $\left(\alpha^{p}, \alpha\right)^{t}$ of $\mathcal{M}^{\prime}=R^{\prime} \otimes_{R} \mathcal{M}$ is fixed by $F$. Thus $\mathcal{M}^{\prime}$ is not simple as an $R^{\prime}[F]$-module.

5.1.1. Examples over the polynomial ring. So far the examples were over a field. Starting with these examples, it is not hard to obtain equivalent examples over higher-dimensional rings. For this let $\left(V, F^{e}\right)$ be the simple unit $K[F]$-module of the last example $\left(K=\mathbb{F}_{p}(x)^{1 / p^{\infty}}\right)$. Let $R$ be a regular $K$-algebra, essentially of finite type over $K$ (e.g., $R=K \otimes_{\mathbb{F}_{p}} \mathbb{F}_{p}\left[x_{1}, \ldots, x_{n}\right]=K\left[x_{1}, \ldots, x_{n}\right]$ ). Let $\mathcal{M}=R \otimes_{K} V$, where we point out that $R$ is a simple $D_{R}$-module and $\operatorname{End}_{D_{R}}(R)=K$. Then $\mathcal{M}$ carries a natural $R\left[F^{e}\right]$-structure defined by $F_{\mathcal{M}}^{e}(n \otimes v)=n^{p^{e}} \otimes F^{e}(v)$. This is exactly the situation of Proposition [3.16, and it follows that the unit $K\left[F^{e}\right]-$ submodules of $V$ are in one-to-one correspondence with the unit $R\left[F^{e}\right]$-submodules 
of $\mathcal{M}$. Thus, the simplicity of $V$ as a unit $K[F]$-module implies that $\mathcal{M}$ is a simple unit $R[F]$-module. Clearly, since $V$ is not $D_{K}$-simple, $\mathcal{M}$ is not $D_{R^{-}}$simple.

\section{REFERENCES}

[1] Pierre Berthelot. D-modules arithmétiques. I. Opérateurs différentiels de niveau fini. Ann. Sci. École Norm. Sup. (4), 29(2):185-272, 1996. MR 97b:14019

[2] Pierre Berthelot. D-modules arithmétiques. II. Descente par Frobenius. Mém. Soc. Math. France (N.S.), 2000. MR 2001k:14043

[3] Manuel Blickle. The intersection homology D-module in finite characteristic. Ph.D. thesis, University of Michigan, 2001. arXiv:math.AG/0110244.

[4] Manuel Blickle. Tight closure and the intersection homology $D$-module in finite characteristic. in preparation, 2002.

[5] Rikard Bøgvad. Some results on $D$-modules on Borel varieties in characteristic $p>0 . J$. Algebra, 173(3):638-667, 1995. MR 97a:14015

[6] Jean Dieudonné. Lie groups and Lie hyperalgebras over a field of characteristic $p>0$. II. Amer. J. Math., 77:218-244, 1955. MR 16:789f

[7] J. Dixmier. Représentationes irréductibles des algèbres de Lie nilpotentes. An. Acad. Brasil, 35:491-519, 1963. MR 32:165

[8] David Eisenbud. Commutative algebra. Springer-Verlag, New York, 1995. MR 97a:13001

[9] Mathew Emerton and Mark Kisin. Riemann-Hilbert correspondence for unit $\mathcal{F}$-crystals. I. in preperation, 1999.

[10] Mathew Emerton and Mark Kisin. Riemann-Hilbert correspondence for unit $\mathcal{F}$-crystals. II. in preperation, 2000.

[11] Burkhard Haastert. Über Differentialoperatoren und d-Moduln in positiver Charakteristik. Manuscripta Math., 58(4):385-415, 1987. MR 88i:14048

[12] Burkhard Haastert. On direct and inverse images of $d$-modules in prime characteristic. Manuscripta Math., 62(3):341-354, 1988. MR 89j:14011

[13] Craig L. Huneke and Rodney Y. Sharp. Bass numbers of local cohomology modules. Trans. Amer. Math. Soc., 339(2):765-779, 1993. MR 93m:13008

[14] Gennady Lyubeznik. Finiteness properties of local cohomology modules (an application of $D$-modules to commutative algebra). Invent. Math., 113(1):41-55, 1993. MR 94e:13032

[15] Gennady Lyubeznik. $\mathcal{F}$-modules: an application to local cohomology and $D$-modules in characteristic $p>0$. Journal für die reine und angewandte Mathematik, 491:65-130, 1997. MR 99c:13005

[16] Gennady Lyubeznik. Finiteness properties of local cohomology modules: a characteristic-free approach. J. Pure Appl. Algebra, 151(1):43-50, 2000. MR 2001g:13038

[17] Gennady Lyubeznik. Injective dimension of $D$-modules: a characteristic-free approach. $J$. Pure Appl. Algebra, 149(2):205-212, 2000. MR 2001g:13029

[18] Daniel Quillen. On the endomorphism ring of a simple module over an enveloping algebra. Proc. Amer. Math. Soc. 21:171-172, 1969. MR 39:252

[19] S. P. Smith. Differential operators on the affine and projective lines in characteristic $p>0$. In Séminaire d'algèbre Paul Dubreil et Marie-Paule Malliavin, 37ème année (Paris, 1985), pages 157-177. Springer, Berlin, 1986. MR 89d:16047

[20] S. P. Smith. The global homological dimension of the ring of differential operators on a nonsingular variety over a field of positive characteristic. J. Algebra, 107(1):98-105, 1987. MR 88b:16045

[21] Amnon Yekutieli. An explicit construction of the Grothendieck residue complex. Astérisque, 208: 1992. With an appendix by Pramathanath Sastry. MR 94e:14026

Universität Essen, FB6 Mathematik, 45117 Essen, Germany

E-mail address: manuel.blickle@uni-essen.de 